\title{
Dynamic analysis of a harmonically excited on-board rotor-bearing system
}

\author{
M Dakel, S Baguet, R Dufour \\ Université de Lyon, CNRS, INSA-Lyon, LaMCoS UMR5259, F-69621, France
}

\begin{abstract}
The aim of this paper is to investigate the dynamic behavior of an on-board rotor mounted on elastic bearings in the presence of rigid support movements. The proposed on-board rotor model is based on the Timoshenko beam finite elements. It takes into account the six deterministic translations and rotations of its rigid support and the geometric asymmetry of shaft and/or rigid disks. Thus the obtained linear equations of motion of the rotating rotor in bending contain time-varying parametric terms which can lead to lateral dynamic instability. The influence of rotational or translational motions of the support is analyzed by means of orbits of the rotor, responses in the time domain and fast Fourier transforms (FFTs).
\end{abstract}

\section{INTRODUCTION}

Many industrial applications include rotating machines and the rotor plays a paramount role in them. There are many studies concerning the prediction of dynamics of rotor systems mounted on elastic bearings in the case of a fixed support $[1,2]$. Some studies observed the instability of systems subjected to parametric excitations [3]. Kang et al. [4] employed the Timoshenko beam finite elements for modeling asymmetric rotor-bearing systems. Some other works concentrated on the behavior of a rotor under seismic excitations [5-7]. Subbiah et al. [8] studied the response of rotor systems under random support excitations using modal analysis methods. Lee et al. [9] focused on the experimental behavior of a rotor under shock support excitation. Da Silva Tuckmantel et al. [10] represented the supporting structure (foundation) of a rotating system by coupled as well as uncoupled modes for calculating the system response. In [11], experimental tests have been shown for a flexibly supported undamped rigid block foundation in rotating machinery. Duchemin et al. [12] observed the stability of a simple rotor model under a sinusoidal support rotation. Driot et al. [13] described the orbits of a rotor induced by a harmonic rotational support movement. El-Saeidy and Sticher [14] obtained the responses of a rigid rotor-bearing system subjected to rotating mass unbalance plus harmonic support excitations. Das et al. [15] investigated the active vibration control of a flexible rotor system excited by mass unbalance and periodic rotational motion of the support. Among all the literature mentioned above, there are references studying support-excited rotor systems and whose few works dealt with the harmonically excited on-board rotors [12-15]. Moreover, these references concentrated on the investigation of dynamic behavior of either simple rotors, rotors supported by elastic bearings with constant damping and stiffness coefficients or rotors excited by simple support motions. As a consequence, the applications proposed in these works are not suitable for realistic ones. In this paper, an improved model is presented. Namely, an asymmetric rotor is discretized using the finite element method based on the Timoshenko beam theory, mounted on hydrodynamic bearings linearized with damping and stiffness coefficients calculated using the Reynolds equation [16], and excited by different motions of its support. The rotary inertia, the gyroscopic inertia, the shear 
deformation of shaft and the geometric asymmetry of the rotor are taken into consideration. The effect of rotor internal damping is not included. The equations of motion point out periodic parametric terms due to the asymmetry of the rotor and time-varying parametric terms due to the rotational support excitations. In the presented application, the rotor mounted on elastic linear bearings is subjected to rotating mass unbalance combined with sinusoidal rotational or translational support motions. Numerical solutions are computed and analyzed by means of rotor orbits, time history responses and fast Fourier transforms (FFTs).

\section{ANALYTICAL MODELING OF AN ON-BOARD ROTOR-BEARING SYSTEM}

\subsection{Preliminary calculations}

Three principal reference frames shown in Figure 1a are introduced to take into account the mobility of the rigid support of the rotor. They are attached to the ground $R^{g}$, the rigid support $R$ and the moving rotor $R^{\prime}$. The rotational motions of the rotor support are defined by the angular velocity vector components $\omega^{x}(t), \omega^{y}(t)$ and $\omega^{z}(t)$ of the rigid support $R$ with respect to the ground $R^{g}$ projected in the frame $R$. The translational motions of the rotor support are defined by the coordinates $x_{O}(t), y_{O}(t)$ and $z_{O}(t)$ of the position vector $\mathbf{O}^{9} \mathbf{O}$ expressed in the frame $R$. The Euler angles $\psi(y, t), \theta(y, t)$ and $\Phi(t)$ (Figure 1b) allow defining the orientation of the rotor $R^{\prime}$ with respect to its support $R$. The angular velocity vector of the rotor $R^{\prime}$ with respect to the ground $R^{g}$ is defined by:

$$
\boldsymbol{\omega}_{R^{\prime}}^{R^{g}}=\boldsymbol{\omega}_{R}^{R^{g}}+\boldsymbol{\omega}_{R^{\prime}}^{R}=\left\langle\omega^{x^{\prime}}, \omega^{y^{\prime}}, \omega^{\omega^{\prime}}\right\rangle_{R^{\prime}}^{T}
$$

where $T$ is a matrix transpose. The components $\left(\omega^{x^{\prime}}, \omega^{y^{\prime}}, \omega^{z^{\prime}}\right)$ are formulated as a function of $(\psi, \theta, \Phi)$ and their time derivative as well as $\left(\omega^{x}, \omega^{y}, \omega^{z}\right)$. The rotor is supposed to rotate at a constant speed $\Omega$. So the spinning angle $\Phi$ is replaced by $\Omega t$ and its derivative $\Phi^{\bullet}$ by $\Omega$ ( $\bullet$ denotes differentiation with respect to time $t$ ). Let us consider a generic point $C^{0}$ along the elastic line of the nondeformed shaft. Its coordinates in the frame $R$ are $(0, y, 0)$. It is interesting to study the translational displacements $u(y, t)$ and $w(y, t)$ of the point $C^{0}$ due to bending expressed respectively with respect to the $O x$ and $O z$ axes of the frame $R$.

(a) $x^{\circ}$

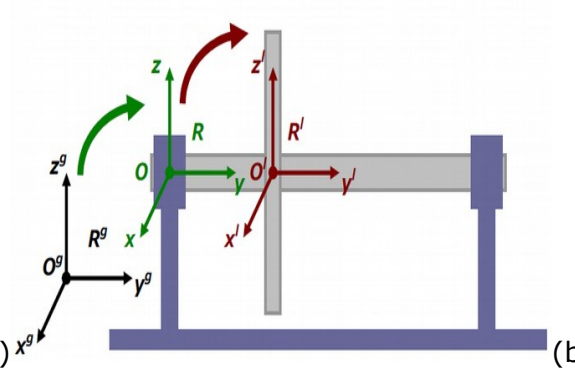

(b) $x$

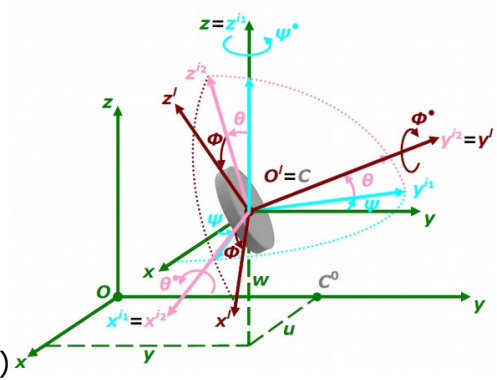

Figure 1. (a) Reference frames for the on-board rotor, (b) Euler angles

\subsection{Energy and virtual work calculations}

\subsubsection{Disk}

Since the disk is considered to be rigid, only its kinetic energy $T_{d}$ is calculated as follows [12]:

$$
T_{d}=\frac{m_{d}}{2}\left(\mathbf{v}_{o^{g}}^{R^{g}}\right)^{2}+\frac{1}{2}\left(\boldsymbol{\omega}_{R^{\prime}}^{R^{g}}\right)^{T} \mathbf{I}_{m_{d}} \boldsymbol{\omega}_{R^{\prime}}^{R^{g}} \quad \text { with } \quad \mathbf{I}_{m_{d}}=\operatorname{diag}\left[I_{m_{d}}^{m o}+I_{m_{d}}^{d i} \quad I_{m_{d}}^{\nu} \quad I_{m_{d}}^{m o}-I_{m_{d}}^{d i}\right]
$$


where $m_{d}$ is the mass of the disk, $\mathbf{v}_{O^{\prime}}^{R^{g}}$ is the translational velocity vector of its center and $\mathbf{I}_{m_{d}}$ is its principal inertia tensor. $I_{m_{d}}^{m o}$ and $I_{m_{d}}^{d i}$ are used to distinguish the effects due to the mean inertia of the disk mass and those due to the inertia modeling the geometric asymmetry of the disk. $I_{m_{d}}^{y}$ is the inertia of the disk mass about the $O y$ axis. The translational velocity vector components $\dot{u}_{O^{\prime}}, \dot{v}_{O^{\prime}}$ and $\dot{w}_{O^{\prime}}$ of the disk center are functions of $\left(\omega^{x}, \omega^{y}, \omega^{z}\right)$ and $\left(x_{0}, y_{0}, z_{0}\right)$. The final expression of the kinetic energy of the disk having a mass center placed at the arbitrary abscissa $y_{d}$ relative to the frame $R$ is given by [12]:

$$
T_{d}=\frac{m_{d}}{2}\left(\dot{u}_{O^{\prime}}^{2}+\dot{v}_{O^{\prime}}^{2}+\dot{W}_{O^{\prime}}^{2}\right)+\frac{1}{2}\left(I_{m_{d}}^{m o}\left(\omega^{x^{\prime} 2}+\omega^{z^{\prime} 2}\right)+I_{m_{d}}^{y} \omega^{y^{\prime} 2}+I_{m_{d}}^{d i}\left(\omega^{x^{\prime} 2}-\omega^{z^{\prime} 2}\right)\right)
$$

\subsubsection{Shaft}

Since the shaft is supposed to be flexible, it is characterized by the kinetic and strain energies and modeled by beam elements. The kinetic energy of a shaft can be obtained by taking an elementary volume of the shaft which can be considered as a disk of thickness $d y$. Thus the expression of the kinetic energy of a shaft has the following form [12]:

$$
T_{s h}=\frac{\rho_{s h} S_{s h}}{2} \int_{0}^{l_{s h}}\left(\dot{u}_{O^{\prime}}^{2}+\dot{v}_{O^{\prime}}^{2}+\dot{w}_{O^{\prime}}^{2}\right) d y+\frac{1}{2}\left(\rho_{s h} I_{S s h}^{m_{s h}} \int_{0}^{l_{s h}}\left(\omega^{x^{\prime} 2}+\omega^{z^{\prime} 2}\right) d y+2 \rho_{s h} I_{S_{s h}}^{m o} \int_{0}^{l_{s h}} \omega^{y^{\prime} 2} d y+\rho_{s h} I_{S_{s h}^{d i}}^{l_{s h}}\left(\omega_{0}^{x^{\prime} 2}-\omega^{z^{\prime 2} 2}\right) d y\right.
$$

where $\rho_{s h}, S_{s h}$ and $I_{s h}$ are respectively the density, the cross-section and the length of the shaft. $I_{S_{s h}}^{m o}$ and $I_{S_{s h}}^{d i}$ are respectively the mean inertia of the cross-section and the inertia characterizing the asymmetry of the shaft. The rigid support motion relative to the ground has no influence on the strain energy of the shaft because the stresses depend only on the transverse deflection of the shaft with respect to the rotor support $R$. In addition to the bending deformation, the shear effects highlighted by Timoshenko and the centrifugal stress due to the support rotations are taken into account. The strain energy of a shaft is:

$$
\begin{aligned}
& U_{s h}=\frac{E_{s h} I_{S_{s h}}^{m o}}{2} \int_{0}^{I_{s h}}\left(\left(\frac{\partial \psi}{\partial y}\right)^{2}+\left(\frac{\partial \theta}{\partial y}\right)^{2}\right) d y+\frac{G_{s h} k_{s h}^{m o} S_{s h}}{2} \int_{0}^{I_{s h}}\left(\left(\frac{\partial u}{\partial y}+\psi\right)^{2}+\left(\frac{\partial W}{\partial y}-\theta\right)^{2}\right) d y-\frac{1}{2}\left(E_{s h} I_{S_{s h}}^{d i} \int_{0}^{l_{s h}}\left(\left(\frac{\partial \psi}{\partial y}\right)^{2}-\left(\frac{\partial \theta}{\partial y}\right)^{2}\right)\right. \\
& \left.-G_{s h} K_{s h}^{d i} S_{s h} \int_{0}^{l_{s h}}\left(\left(\frac{\partial u}{\partial y}+\psi\right)^{2}-\left(\frac{\partial W}{\partial y}-\theta\right)^{2}\right) d y\right) \cos (2 \Omega t)-\left(E_{s h} I_{S_{s h}}^{d i} \int_{0}^{l_{s h}} \frac{\partial \psi}{\partial y} \frac{\partial \theta}{\partial y} d y\right. \\
& \left.-G_{s h} k_{s h}^{d i} S_{s h} \int_{0}^{l_{s h}}\left(\frac{\partial u}{\partial y}+\psi\right)\left(\frac{\partial w}{\partial y}-\theta\right) d y\right) \sin (2 \Omega t)+\frac{\rho_{s h} S_{s h}}{4} \int_{0}^{l_{s h}}\left(l_{s h}^{2}-y^{2}\right)\left(\left(\frac{\partial u}{\partial y}\right)^{2}+\left(\frac{\partial w}{\partial y}\right)^{2}\right) d y\left(\omega^{\times 2}+\omega^{22}\right)
\end{aligned}
$$

where $E_{s h}$ and $G_{s h}$ are respectively the Young's modulus and the shear modulus of the shaft $\left(G_{s h}=E_{s h} /\left(2\left(1+v_{s h}\right)\right)\right.$ hence $V_{s h}$ is the Poisson's ratio $) . k_{s h}^{m o}$ and $k_{s h}^{d i}$ are respectively the mean shear coefficient and the shear coefficient relative to the section asymmetry of the shaft.

\subsubsection{Mass unbalance}

Let us consider a concentrated mass unbalance $m_{m u}$ positioned at a point $P_{m u}$ of the disk $\left(y_{m u}=y_{d}\right)$ with a distance $r_{m u}$ from the geometric center of the shaft. Its initial angle with the $O z$ axis of the frame $R$ at rest is $\eta_{m u}$. The components of the mass unbalance translational velocity vector are functions of $\left(\omega^{x}, \omega^{y}, \omega^{z}\right)$ and $\left(x_{0}, y_{0}, z_{0}\right)$ and are used in the kinetic energy which characterizes the mass unbalance [12]:

$$
T_{m u}=\frac{m_{m u}}{2}\left(\mathbf{v}_{P_{m u}}^{R^{g}}\right)^{2}
$$




\subsubsection{Bearing}

Figure 2 a shows a simple diagram of a hydrodynamic bearing which is composed of a fixed journal containing a rotating shaft. The points $O$ and $O^{\prime}=C_{\text {sep }}$ represent respectively the bearing center and the shaft center. The radius, length and clearance of the bearing are respectively $r_{b e}, l_{b e}$ and $c_{b e}=r_{b e}-r_{s h}$ where $r_{s h}$ is the shaft radius. At a constant rotor speed $\Omega$ and for a constant static load $W_{r}$ created by the rotor weight, the shaft center $C_{\text {sep }}$ in the bearing holds a static equilibrium position defined by the vector $\boldsymbol{\delta}_{b e, s e p}=\left\langle u_{b e, s e p}, w_{b e, s e p}\right\rangle_{R}^{T}$ expressed in the frame $R$ or equivalently by the eccentricity $e_{b e}=\left\|\boldsymbol{\delta}_{b e, s e p}\right\|$ of the shaft center in the journal and the attitude angle $\varphi_{b e}$ between the $W_{r}$ load direction and the line of centers $\mathbf{O C}_{\text {sep }}$. In the present study, the short bearing theory is considered $\left(I_{b e} / d_{b e} \leq 1 / 8\right.$ where $\left.d_{b e}=2 r_{b e}\right)$ and the static solution can be obtained using the following formulations deduced from the Reynolds equation with the Gümbel boundary conditions [16]:

$$
W_{r}=\mu r_{b e}\left(\frac{r_{b e}}{c_{b e}}\right)^{2}\left(\frac{l_{b e}}{d_{b e}}\right)^{2} I_{b e} \Omega \frac{\varepsilon_{b e}\left(16 \varepsilon_{b e}^{2}+\Pi^{2}\left(1-\varepsilon_{b e}^{2}\right)\right)^{\frac{1}{2}}}{\left(1-\varepsilon_{b e}^{2}\right)^{2}} \text { and } \tan \left(\varphi_{b e}\right)=\frac{\pi}{4} \frac{\sqrt{1-\varepsilon_{b e}^{2}}}{\varepsilon_{b e}}
$$

where $\varepsilon_{b e}$ is the relative eccentricity $\left(\varepsilon_{b e}=e_{b e} / c_{b e}\right)$ and $\mu$ is the fluid film dynamic viscosity. The above nonlinear equation is solved by an iterative Newton-Raphson method and provides the eccentricity $\varepsilon_{b e}$ and then the static radial displacement $e_{b e}$ of the shaft center. The vector $\boldsymbol{\delta}_{\text {be, sep }}$ is obtained by a classical change of basis. The hydrodynamic fluid forces $\mathbf{F}_{b e}=\left\langle F_{b e}{ }^{u}, F_{b e}{ }^{w}\right\rangle_{R}{ }^{T}$ produced by the bearings and expressed in the frame $R$ can be obtained by integration of the fluid film pressure (Reynolds equation in the dynamic regime) over the bearing. In order to apply the Lagrange's equations, the virtual work $\delta W_{b e}$ of these forces has to be established:

$$
\delta W_{b e}=\mathbf{F}_{b e}^{T}\left(\mathbf{\delta}_{b e}, \dot{\boldsymbol{\delta}}_{b e}\right) \delta \mathbf{\delta}_{b e}
$$

If the lateral dynamic displacements $\boldsymbol{\delta}_{b e}=\left\langle u_{b e}, w_{b e}\right\rangle_{R}{ }^{T}$ of the shaft elastic line are assumed to be small in the vicinity of the static position $\boldsymbol{\delta}_{b e, s e p}$, the linear analysis can be used by finding the first order Taylor series of the dynamic fluid film forces:

$$
\mathbf{F}_{b e}\left(\boldsymbol{\delta}_{b e}, \dot{\boldsymbol{\delta}}_{b e}\right)=\mathbf{F}_{b e}\left(\boldsymbol{\delta}_{b e, s e p}, \mathbf{0}\right)-\mathbf{c}_{b e} \Delta \dot{\mathbf{\delta}}_{b e}-\mathbf{k}_{b e} \Delta \boldsymbol{\delta}_{b e}
$$

with $\mathbf{c}_{b e}=\left[\begin{array}{ll}c_{b e}^{x x} & c_{b e}^{x z} \\ c_{b e}^{z x} & c_{b e}^{z z}\end{array}\right], \mathbf{k}_{b e}=\left[\begin{array}{ll}k_{b e}^{x x} & k_{b e}^{x z} \\ k_{b e}^{z x} & k_{b e}^{z z}\end{array}\right], \quad \Delta \boldsymbol{\delta}_{b e}=\boldsymbol{\delta}_{b e}-\boldsymbol{\delta}_{b e, \text { sep }}$ and $\boldsymbol{\Delta} \dot{\boldsymbol{\delta}}_{b e}=\dot{\boldsymbol{\delta}}_{b e} \cdot \mathbf{c}_{b e}$ and $\mathbf{k}_{b e}$ are the damping and stiffness matrices of the linearized hydrodynamic bearing (Figure 2b) whose analytical expressions can be found in [16].

(a)

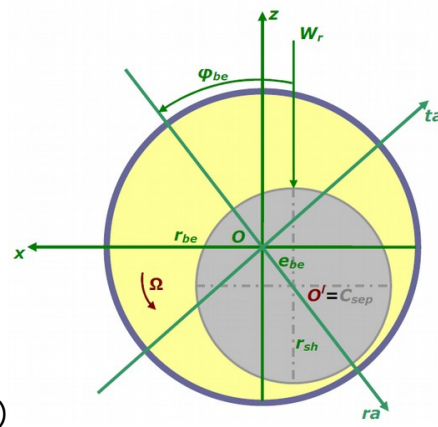

(b)

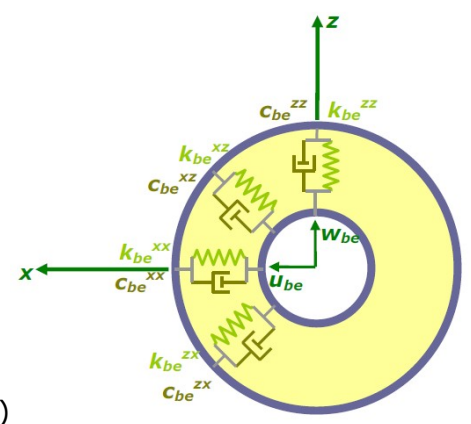

(b) Bearing damping and stiffness

\section{Figure 2. (a) Hydrodynamic bearing, (b)
coefficients}




\subsection{Equations of motion}

The finite element method is chosen for discretizing the rotor and describing its flexural motion as a function of the nodal displacement vector defined by $\boldsymbol{\delta}^{n}=\left\langle u^{n}, w^{n}, \theta^{n}, \psi^{n}\right\rangle_{R}{ }^{T}$, i.e., the rotor has two translations and two rotations at each node. The finite element used for the shaft modeling has two nodes and the shape functions are based on the Timoshenko beam theory. The effect of rotor internal damping is neglected. The linear equations of motion of the finite element rotor model are obtained after applying the Lagrange's equations to the energies for the disk, the shaft finite element and the mass unbalance as well as to the virtual work of the hydrodynamic bearings and assembling appropriately the produced vectors and matrices. They are written with respect to the noninertial reference frame connected to the rigid support $R$ of the rotor:

$$
\begin{aligned}
& \mathbf{M}_{r}(t) \ddot{\boldsymbol{\delta}}_{r}+\mathbf{C}_{r}(t) \dot{\boldsymbol{\delta}}_{r}+\mathbf{K}_{r}(t) \boldsymbol{\delta}_{r}=\mathbf{F}_{r}(t)+\mathbf{F}_{b e}\left(\boldsymbol{\delta}_{r, \text { sep }}, \mathbf{0}\right)+\mathbf{K}_{b e} \boldsymbol{\delta}_{r, \text { sep }} \\
& \mathbf{M}_{r}(t)=\mathbf{M}_{d, s h}+\mathbf{M}_{d, s h}^{c} \cos (2 \Omega t)+\mathbf{M}_{d, s h}^{s} \sin (2 \Omega t) \\
& \mathbf{C}_{r}(t)=\mathbf{C}_{b e}+\mathbf{C}_{d, s h}^{g} \Omega+\mathbf{C}_{d, s h}^{g, c} \Omega \cos (2 \Omega t)+\mathbf{C}_{d, s h}^{g, s} \Omega \sin (2 \Omega t)+\mathbf{C}_{d, s h, s u}^{r e, \omega^{y}} \omega^{y} \\
& \mathbf{K}_{r}(t)=\mathbf{K}_{b e}+\mathbf{K}_{s h}^{e}+\mathbf{K}_{s h}^{e, c} \cos (2 \Omega t)+\mathbf{K}_{s h}^{e, s} \sin (2 \Omega t)+\mathbf{K}_{d, s h, s u}^{r e, \omega^{y}} \dot{\omega}^{y}+\mathbf{K}_{d, s h, s u}^{r e, s \omega^{y}} \Omega \omega^{y} \\
& +\left(\mathbf{K}_{d, s h, s u}^{r e, \omega^{\times 2}}+\mathbf{K}_{d, s h, s u}^{g s e, \omega^{x 2}}\right) \omega^{\times 2}+\mathbf{K}_{d, s h, s u}^{r e, \omega^{22}} \omega^{y 2}+\left(\mathbf{K}_{d, s h, s u}^{r e, \omega^{22}}+\mathbf{K}_{d, s h, s u}^{g s e, \omega^{22}}\right) \omega^{22}+\mathbf{K}_{d, s h, s u}^{r e, \omega^{x}} \omega^{x} \omega^{x} \\
& +\left(\mathbf{K}_{d, s h, s u}^{r e, \omega^{y}, c} \dot{\omega}^{y}+\mathbf{K}_{d, s h, s u}^{r e, s \omega^{y}, c} \Omega \omega^{y}+\mathbf{K}_{d, s h, s u}^{r e, \omega^{x,}, c} \omega^{x 2}+\mathbf{K}_{d, s h, s u}^{r e, \omega^{y}, c} \omega^{y 2}+\mathbf{K}_{d, s h, s u}^{r e, \omega^{22}, c} \omega^{22}\right. \\
& \left.+\mathbf{K}_{d, s h, s u}^{r e, \omega^{x} \omega^{2}, c} \omega^{x} \omega^{z}\right) \cos (2 \Omega t)+\left(\mathbf{K}_{d, s h, s u}^{r e, \omega^{y}, s} \dot{\omega}^{y}+\mathbf{K}_{d, s h, s u}^{r e, \Omega \omega^{y}, s} \Omega \omega^{y}+\mathbf{K}_{d, s h, s u}^{r e, \omega^{x 2}, s} \omega^{x 2}\right. \\
& \left.+\mathbf{K}_{d, s h, s u}^{r e, \omega^{\nu^{2}}, s} \omega^{y 2}+\mathbf{K}_{d, s h, s u}^{r e, \omega^{x} \omega^{2}, s} \omega^{x} \omega^{z}\right) \sin (2 \Omega t) \\
& \mathbf{F}_{r}(t)=\mathbf{F}_{d, s h}^{w_{r}}+\mathbf{F}_{m u}(t)+\mathbf{F}_{m u, s u}(t)+\mathbf{F}_{d, s h, s u}(t)+\mathbf{F}_{d, s h, s u}^{c}(t) \cos (2 \Omega t)+\mathbf{F}_{d, s h, s u}^{s}(t) \sin (2 \Omega t) \\
& =-\mathbf{V}_{d, s h}^{W_{r}} W_{r}+\mathbf{V}_{m u}^{c} \Omega^{2} \cos (\Omega t)+\mathbf{V}_{m u}^{s} \Omega^{2} \sin (\Omega t)+\left(\mathbf{V}_{m u, s u}^{\omega^{y}, c} \dot{\omega}^{y}+\mathbf{V}_{m u, s u}^{\Omega \omega \omega^{y}, c} \Omega \omega^{y}+\mathbf{V}_{m u, s u}^{\omega^{x 2}, c} \omega^{\times 2}\right. \\
& \left.+\mathbf{V}_{m u, s u}^{\omega^{\gamma 2}, c} \omega^{y 2}+\mathbf{V}_{m u, s u}^{\omega^{22}, c} \omega^{22}+\mathbf{V}_{m u, s u}^{\omega^{x} \omega^{2}, c} \omega^{x} \omega^{z}\right) \cos (\Omega t)+\left(\mathbf{V}_{m u, s u}^{\omega^{y}, s} \dot{\omega}^{y}+\mathbf{V}_{m u, s u}^{\Omega \omega^{y}, s} \Omega \omega^{y}\right. \\
& \left.+\mathbf{V}_{m u, s u}^{\omega^{x 2}, s} \omega^{x 2}+\mathbf{V}_{m u, s u}^{\omega^{\gamma 2}, s} \omega^{y 2}+\mathbf{V}_{m u, s u}^{\omega^{22}, s} \omega^{22}+\mathbf{V}_{m u, s u}^{\omega^{x} \omega^{2}, s} \omega^{x} \omega^{z}\right) \sin (\Omega t) \\
& -\mathbf{V}_{d, s h, s u}^{u}\left(\ddot{x}_{O}+2 \dot{z}_{O} \omega^{y}-2 \dot{y}_{O} \omega^{z}+z_{O}\left(\dot{\omega}^{y}+\omega^{x} \omega^{z}\right)-y_{O}\left(\dot{\omega}^{z}-\omega^{x} \omega^{y}\right)-x_{O}\left(\omega^{y 2}+\omega^{z 2}\right)\right) \\
& -\mathbf{V}_{d, s h, s u}^{w}\left(\ddot{z}_{O}+2 \dot{y}_{O} \omega^{x}-2 \dot{x}_{O} \omega^{y}+y_{O}\left(\dot{\omega}^{x}+\omega^{y} \omega^{z}\right)-x_{0}\left(\dot{\omega}^{y}-\omega^{x} \omega^{z}\right)-z_{0}\left(\omega^{x 2}+\omega^{y 2}\right)\right) \\
& -\mathbf{V}_{d, s h, s u}^{y \omega}\left(\dot{\omega}^{x}+\omega^{y} \omega^{z}\right)+\mathbf{V}_{d, s h, s u}^{y u}\left(\dot{\omega}^{z}-\omega^{x} \omega^{y}\right)-\mathbf{V}_{d, s h, s u}^{\theta, m o}\left(\dot{\omega}^{x}+\omega^{y} \omega^{z}\right) \\
& -\mathbf{V}_{d, s h, s u}^{\psi, m o}\left(\dot{\omega}^{z}-\omega^{x} \omega^{y}\right)-\mathbf{V}_{d, s h, s u}^{y \psi}\left(\Omega \omega^{x}+\omega^{x} \omega^{y}\right)+\mathbf{V}_{d, s h, s u}^{y \theta}\left(\Omega \omega^{z}+\omega^{y} \omega^{z}\right) \\
& -\mathbf{V}_{d, s h, s u}^{\theta, d i}\left(\dot{\omega}^{x}-2 \Omega \omega^{z}-\omega^{y} \omega^{z}\right) \cos (2 \Omega t)+\mathbf{V}_{d, s h, s u}^{y, d i}\left(\dot{\omega}^{z}+2 \Omega \omega^{x}+\omega^{x} \omega^{y}\right) \cos (2 \Omega t) \\
& +\mathbf{V}_{d, s h, s u}^{\psi, d i}\left(\dot{\omega}^{x}-2 \Omega \omega^{z}-\omega^{y} \omega^{z}\right) \sin (2 \Omega t)+\mathbf{V}_{d, s h, s u}^{\theta, d i}\left(\dot{\omega}^{z}+2 \Omega \omega^{x}+\omega^{x} \omega^{y}\right) \sin (2 \Omega t)
\end{aligned}
$$

where $\ddot{\boldsymbol{\delta}}_{r}, \dot{\boldsymbol{\delta}}_{r}$ and $\boldsymbol{\delta}_{r}$ are respectively the acceleration, velocity and displacement vectors of the on-board rotor-bearing system of dimension $4\left(n_{\text {esh }}+1\right) \times 1$ hence $n_{\text {esh }}$ is the number of shaft finite elements. $\mathbf{M}_{r}(t), \mathbf{C}_{r}(t)$ and $\mathbf{K}_{r}(t)$ are respectively the parametric mass, damping and stiffness matrices due to the asymmetry of the rotor and to the rotational motions of its support. $\mathbf{F}_{r}(t)$ is the external force vector. $\mathbf{F}_{b e}\left(\boldsymbol{\delta}_{r, s e p}, \mathbf{0}\right)$ is the hydrodynamic force vector caused by the bearings in the static case. This vector is opposite and equal to the rotor weight vector $\mathbf{F}_{d, \text { sh }}^{w_{r}}$. $\boldsymbol{\delta}_{r, \text { sep }}$ is the static solution vector of the rotor due to the hydrodynamic bearing. The subscripts " $d$ ", "sh", "be" and "su" refer respectively to the disk, shaft, bearing as well as support and express the contribution to the phenomenon represented by the corresponding matrix. The superscript " $g$ " refers to the gyroscopic effect, " $e$ " signifies the shaft elasticity corresponding to the bending and shear deformations, "re" is the rotational effects due to the support rotations (these effects come from the kinetic energies of the disk and the shaft) and " $g s e^{\prime \prime}$ is the geometric stiffening effects corresponding to the centrifugal stress due to the support rotations. $\mathbf{V}_{\text {mu, }}$ $\mathbf{V}_{d, s h, s u}$ and $\mathbf{V}_{m u, s u}$ are load vectors associated respectively with the mass unbalance, the inertia force due to support motions and that due to coupling between both 
phenomena. The superscripts $u, w, \psi$ and $\theta$ denote the direction of the action force components associated with the motions of the rotor support. The transient dynamic motion of the rotor is then obtained by solving Equation 10 by means of the implicit Newmark scheme based on the average acceleration. The static equilibrium position $\boldsymbol{\delta}_{r, s e p}$ is used to initialize the transient dynamic problem. The final integration time is chosen such that the transient effects have disappeared and the steady-state regime has been reached.

\section{NUMERICAL EXAMPLES AND DISCUSSION}

The symmetric rotor-linearized hydrodynamic bearing system presented in Figure 3 is subjected to rotating mass unbalance combined with sinusoidal rotational or translational support motions: the rotation around the $O x$ axis is given by $\omega^{x}=\omega^{x, a} \cos \left(\Omega^{x} t\right)$ in $\mathrm{rad} / \mathrm{s}$, while the translation along the $O z$ axis is expressed as $z_{0}=Z_{O} \cos \left(\Omega^{z} t\right)$ in $\mathrm{m}$. The physical properties as well as the geometry of the rotor and the bearings are listed in Table 1 . The shaft is discretized into eight identical 2-node Timoshenko beam finite elements. The disk is located at node 5 and bearings \# 1 and \# 2 are respectively placed at nodes 1 and 9 . The rotor runs at a speed of rotation $\Omega=1200 \mathrm{rpm}$ ( $=20 \mathrm{~Hz}=$ mass unbalance frequency) and its first four natural frequencies are $33.57 \mathrm{~Hz}, 52.7 \mathrm{~Hz}, 542.4 \mathrm{~Hz}$ and $1647.2 \mathrm{~Hz}$. The relative static equilibrium position of the geometric center of the shaft in the fluid film bearings is given by $\boldsymbol{\delta}_{b e, s e p} / c_{b e}=<-0.2855,-0.8789>_{R}{ }^{T}$. The bearing damping and stiffness matrices are expressed in what follows:

$$
\mathbf{c}_{b e}=\left[\begin{array}{ll}
3.50 \times 10^{3} & 1.08 \times 10^{4} \\
1.08 \times 10^{4} & 7.57 \times 10^{4}
\end{array}\right] \mathrm{N} / \mathrm{m} / \mathrm{s} \quad ; \quad \mathbf{k}_{b e}=\left[\begin{array}{ll}
1.30 \times 10^{6} & 1.32 \times 10^{6} \\
6.30 \times 10^{6} & 1.94 \times 10^{7}
\end{array}\right] \mathrm{N} / \mathrm{m}
$$

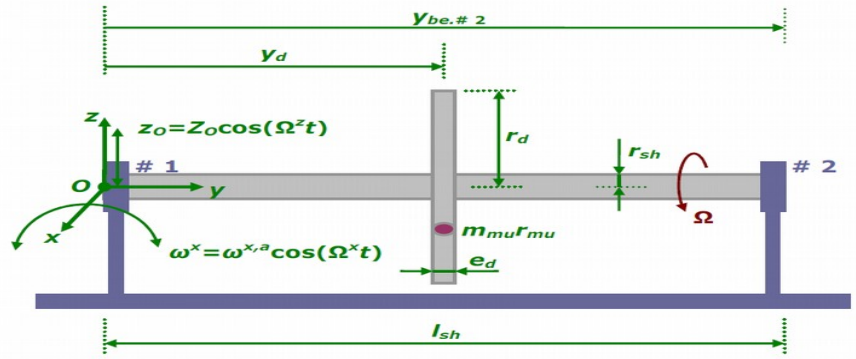

Figure 3. Schematic diagram of the investigated rotor-bearing system

Table 1. Main characteristics of the investigated rotor and the bearings

Density of both disk and shaft material

Radius, thickness and location of the

disk

shaft material

Radius and length of the shaft

Mass unbalance

Radius and length of the bearings

Locations of the bearings

Radial clearance of the bearings

Oil film dynamic viscosity

$$
\begin{array}{r}
\rho_{d}=\rho_{s h}=7800 \mathrm{~kg} / \mathrm{m}^{3} \\
r_{d}=0.15 \mathrm{~m}, e_{d}=0.03 \mathrm{~m}, y_{d}=0.2 \mathrm{~m} \\
E_{s h}=2 \times 10^{11} \mathrm{~N} / \mathrm{m}^{2}, v_{s h}=0.3 \\
r_{s h}=0.04 \mathrm{~m}, I_{s h}=0.4 \mathrm{~m} \\
m_{m u} r_{m u}=1500 \mathrm{~g} \mathrm{~mm}, \eta_{m u}=0^{\circ} \\
r_{b e}=0.04 \mathrm{~m}, l_{b e}=0.01 \mathrm{~m} \\
y_{b e . \# 1}=0 \mathrm{~m}, y_{b e . \# 2}=0.4 \mathrm{~m} \\
c_{b e}=2 \times 10^{-4} \mathrm{~m} \\
\mu=288 \times 10^{-4} \mathrm{~Pa} \mathrm{~s}
\end{array}
$$

All the presented orbits are dimensionless with respect to the bearing clearance $c_{b e}$ and are thus plotted in a unit circle. These orbits show the transient motion within the bearings from the static position $C_{\text {sep }}$ to the steady-state solution. Figure $4 a$ presents a general view of the rotor orbit at the hydrodynamic bearings due to the 
mass unbalance effect. Since the disk is positioned at the middle of the shaft, the orbits at both bearings are identical in the case of a fixed support. The nonsymmetric damping and stiffness coefficients make the bearings anisotropic and the orbit elliptical with diagonal axes defining the phase between the mass unbalance excitation and the rotor response (Figure 4b).

(a)

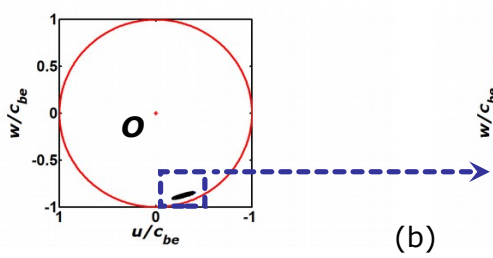

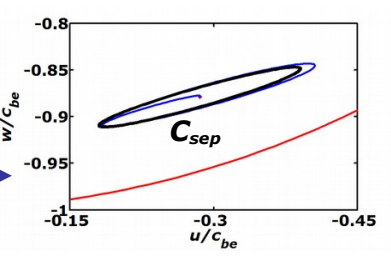

Figure 4. (a) Mass unbalance orbit of the investigated rotor at the bearings for a fixed support, (b) zoom on the orbit

Figures 5 and 6 display the rotor orbits at bearing \# 2 during the harmonic translational or rotational motions of the support. Even though the disk location is symmetric with respect to the bearings, the orbits are not identical in the case of a harmonic support rotation because of its normal and tangential accelerations. Focus is put on bearing \# 2 which is more affected by the support rotation. The orbit characteristics (shape and magnitude) change with the amplitude $\left(z_{0}\right.$ or $\left.\omega^{x, a}\right)$ and frequency $\left(\Omega^{z}\right.$ or $\left.\Omega^{x}\right)$ of the harmonic support excitation. As expected, in the case of very small amplitudes and frequencies of the support motions equal to the mass unbalance frequency, the orbits are similar to those produced when the support is fixed. Increasing the frequencies makes the orbit shapes more complicated; see Figures $5 \mathrm{a}$ and $6 \mathrm{a}$. The dynamic behavior is all the more modified as the frequency of the support excitation is high compared with the natural frequencies or the speed of rotation of the rotor. On the other hand, the amplitudes of the support motion change the $x$ and $z$ orbit magnitudes.

(a) $Z_{o}=10^{-5} \mathrm{~m}$
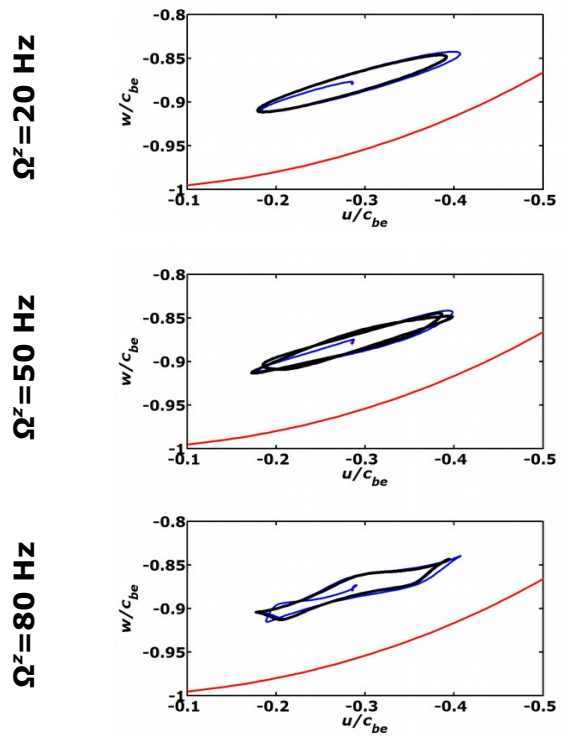

(b) $Z_{o}=5 \times 10^{-5} \mathrm{~m}$
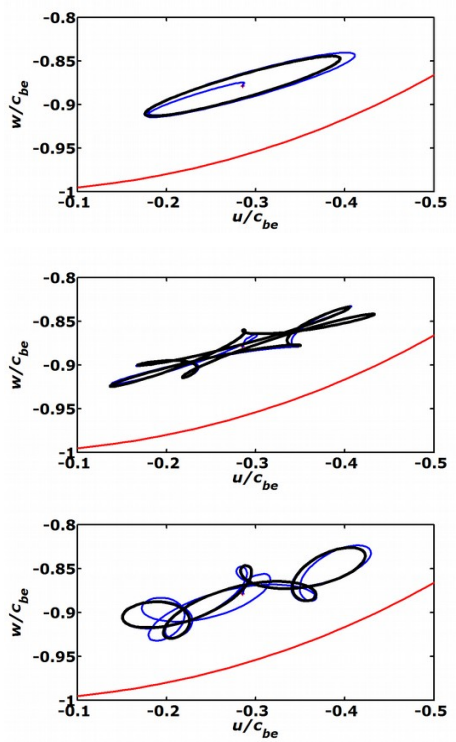

Figure 5. Relative orbits of the rotor at bearing \# 2 for two amplitudes of sinusoidal support translations: (a) $Z_{o}=10^{-5} \mathrm{~m}$, (b) $Z_{o}=5 \times 10^{-5} \mathrm{~m}$ 
(a) $\omega^{x, a}=10^{-2} \mathrm{rad} / \mathrm{s}$
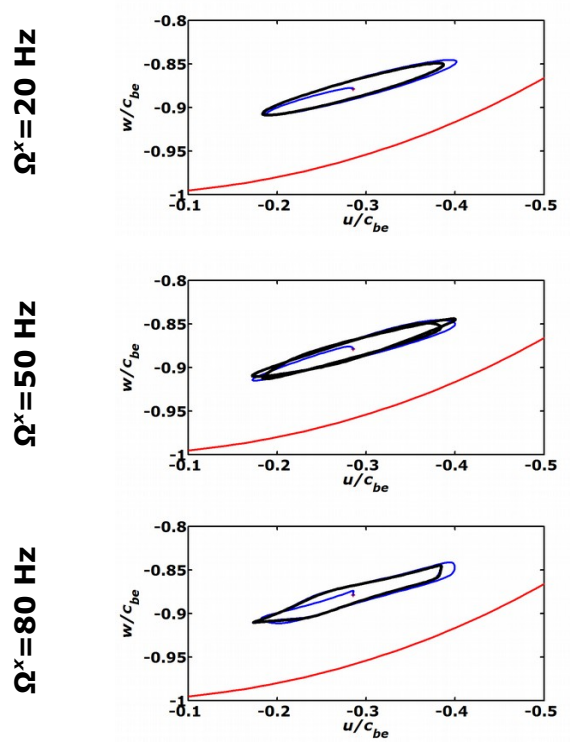

(b) $\omega^{x, a}=5 \times 10^{-2} \mathrm{rad} / \mathrm{s}$
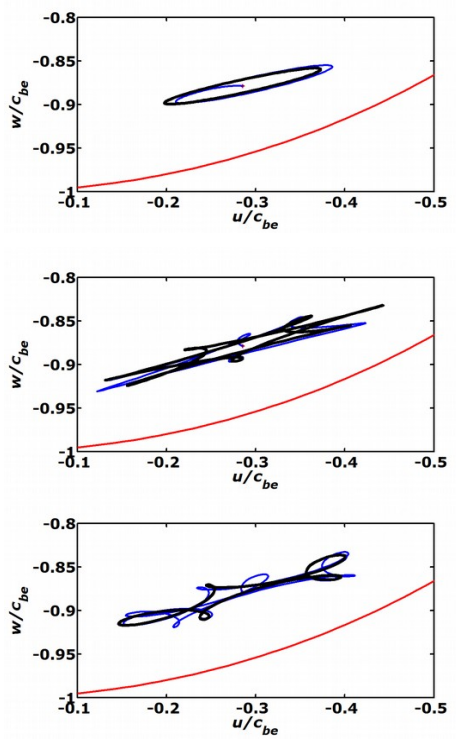

Figure 6. Relative orbits of the rotor at bearing \# 2 for two amplitudes of sinusoidal support rotations: (a) $\omega^{x, a}=10^{-2} \mathrm{rad} / \mathrm{s},(\mathrm{b}) \omega^{x, a}=5 \times 10^{-2} \mathrm{rad} / \mathrm{s}$

The time history responses and their FFTs presented in Figures 7 and 8 exhibit two frequency components due to the mass unbalance excitation $(20 \mathrm{~Hz})$ and to the harmonic support motions $(50 \mathrm{~Hz}$ and $80 \mathrm{~Hz}$ ). The mass unbalance excitation in Equation 12 and thereby the corresponding rotor response are not affected by the support translations. This result is confirmed in Figures $7 \mathrm{~b}$ and $7 \mathrm{c}$ where the FFT peaks at a frequency of $20 \mathrm{~Hz}$ in both $\mathrm{Ox}$ and $\mathrm{Oz}$ directions have exactly the same displacement amplitude for support translations of frequency $50 \mathrm{~Hz}$ and $80 \mathrm{~Hz}$. Even if the support rotations have an influence on the mass unbalance excitation (see Equation 12), this influence does not appear clearly in the FFTs of the rotor motion during the harmonic support rotation because its amplitude is very small compared to the speed of rotation of the rotor (mass unbalance frequency).

\section{CONCLUSIONS}

A finite element model is presented to analyze the dynamic behavior of a symmetric on-board rotor whose support is subjected to sinusoidal rotation or translation. The rotational effects and the geometric stiffening effects relative to the centrifugal stressing due to the support rotations are taken into account. The support rotations create time-varying parametric coefficients which can lead to lateral dynamic instability. It is noted that the shape and the magnitude of the rotor orbits can be significantly affected by the frequency and amplitude of the support motion respectively. It is shown that the mass unbalance forces and also the corresponding responses can depend on the support rotation around a transverse axis (in the presence of considerable rotation compared to the speed of rotation of the rotor) but not on the support translation along a lateral direction. The two frequency components due to the mass unbalance excitation and to the harmonic support motions appear in the time history responses and the FFTs of the rotor oscillations. In the case of large orbits, the assumption of a linearized hydrodynamic bearing model is questionable. A nonlinear bearing model is to be 
considered. Future work will concern the dynamics of such a nonlinear on-board rotor-bearing system.

Displacement in the $O x$ direction
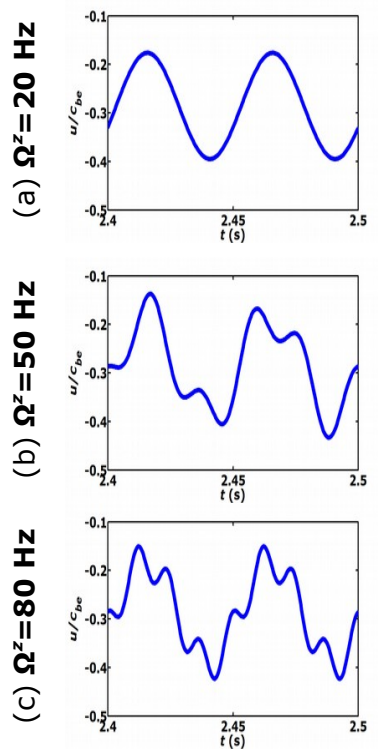
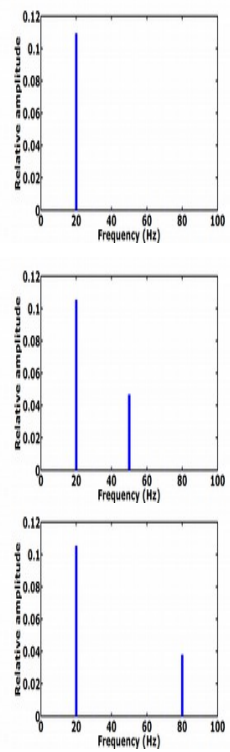

Displacement in the $\mathrm{Oz}$ direction
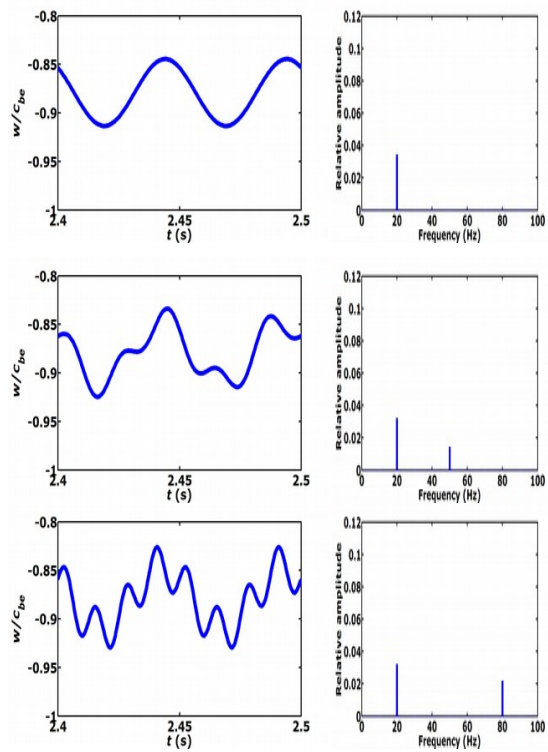

Figure 7. Relative responses and FFTs of the lateral vibrations at bearing \# 2 for support translations of amplitude $Z_{o}=5 \times 10^{-5} \mathrm{~m}$ and frequency: (a) $\Omega^{z}=20 \mathrm{~Hz}$, (b) $\Omega^{z}=50 \mathrm{~Hz}$, (c) $\Omega^{z}=80 \mathrm{~Hz}$

\section{Displacement in the $O x$ direction}
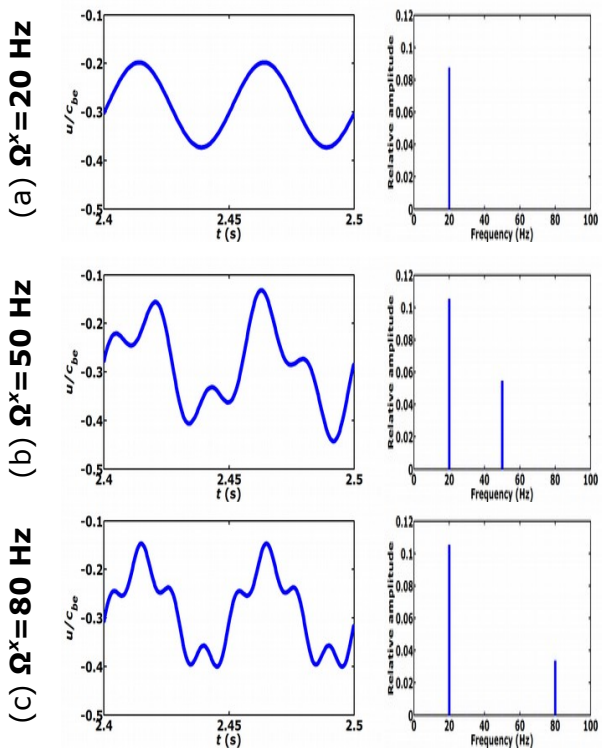

Displacement in the $\mathrm{Oz}$ direction
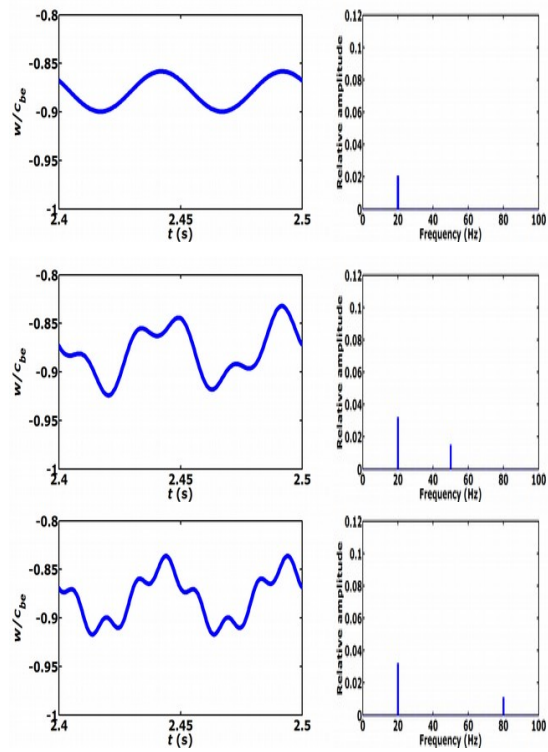

Figure 8. Relative responses and FFTs of the transverse vibrations at bearing \# 2 for support rotations of amplitude $\omega^{x, a}=5 \times 10^{-2} \mathrm{rad} / \mathrm{s}$ and frequency: (a) $\Omega^{x}=20 \mathrm{~Hz}$, (b) $\Omega^{x}=50 \mathrm{~Hz}$, (c) $\Omega^{x}=80 \mathrm{~Hz}$ 


\section{REFERENCE LIST}

[1] Lalanne, M., Ferraris, G., 1998. Rotordynamics Prediction in Engineering. Wiley, Chichester.

[2] Genta, G., 2005. Dynamics of Rotating Systems. Springer, New York.

[3] Dufour, R., Berlioz, A., 1998. Parametric instability of a beam due to axial excitations and to boundary conditions. ASME Journal of Vibration and Acoustics, 120(2), pp. 461-467.

[4] Kang, Y., Shih, Y.-P., Lee, A.-C., 1992. Investigation on the steady-state responses of asymmetric rotors. ASME Journal of Vibration and Acoustics, 114(2), pp. 194-208.

[5] Srinivasan, V., Soni, A., 1984. Seismic analysis of a rotor-bearing system. Earthquake Engineering and Structural Dynamics, 12(3), pp. 287-311.

[6] Hori, Y., Kato, T., 1990. Earthquake-induced instability of a rotor supported by oil film bearings. ASME Journal of Vibration and Acoustics, 112(2), pp. $160-165$.

[7] Suarez, L.E., Singh, M.P., Rohanimanesh, M.S., 1992. Seismic response of rotating machines. Earthquake Engineering and Structural Dynamics, 21(1), pp. 21-36.

[8] Subbiah, R., Bhat, R.B., Sankar, T.S., 1985. Response of rotors subjected to random support excitations. ASME Journal of Vibration, Acoustics, Stress and Reliability in Design, 107(4), pp. 453-459.

[9] Lee, A.S., Kim, B.O., Kim, Y.-C., 2006. A finite element transient response analysis method of a rotor-bearing system to base shock excitations using the state-space Newmark scheme and comparisons with experiments. Journal of Sound and Vibration, 297(3-5), pp. 595-615.

[10] Da Silva Tuckmantel, F.W., Cavalca, K.L., de Castro, H.F., Felscher, P., Markert, R., 2011. An analysis on the supporting structure representative model in rotating systems. Proceedings of the Tenth International Conference on Vibration Problems, Prague, Czech Republic, 5-8 September.

[11] Feng, N., Hahn, E., 2011. Rotor-model-based identification of foundations in rotating machinery using modal parameters. Proceedings of the Tenth International Conference on Vibration Problems, Prague, Czech Republic, 5-8 September.

[12] Duchemin, M., Berlioz, A., Ferraris, G., 2006. Dynamic behavior and stability of a rotor under base excitation. ASME Journal of Vibration and Acoustics, 128(5), pp. 576-585.

[13] Driot, N., Lamarque, C.H., Berlioz, A., 2006. Theoretical and experimental analysis of a base-excited rotor. ASME Journal of Computational and Nonlinear Dynamics, 1(3), pp. 257-263.

[14] El-Saeidy, F.M.A., Sticher, F., 2010. Dynamics of a rigid rotor linear/nonlinear bearings system subject to rotating unbalance and base excitations. Journal of Vibration and Control, 16(3), pp. 403-438.

[15] Das, A.S., Dutt, J.K., Ray, K., 2010. Active vibration control of unbalanced flexible rotor-shaft systems parametrically excited due to base motion. Applied Mathematical Modelling, 34(9), pp. 2353-2369.

[16] Frêne, J., Nicolas, D., Degueurce, B., Berthe, D., Godet, M., 1997. Hydrodynamic Lubrication Bearings and Thrust Bearings. Elsevier Science, Amsterdam. 